\title{
Prevalence of multidrug efflux pump requiring ciprofloxacin, ofloxacin and pefloxacin as substrates, among clinical isolates of Pseudomonas aeruginosa
}

\author{
Omoregie, R., ${ }^{*}{ }^{1,2}$ Airueghionmon, D.J.U., ${ }^{1}$ Okonkwo, J.O., ${ }^{2}$ Airueghionmon, U.- E., ${ }^{1}$ Ibeh, I.N. ${ }^{3}$ and Ogefere, H.O \\ ${ }^{1}$ School of Medical Laboratory Sciences, University of Benin Teaching Hospital, P.M.B. 1111, Benin City, Edo \\ State, Nigeria. \\ ${ }^{2}$ Department of Pharmaceutical Microbiology, Faculty of Pharmacy, University of Benin, Benin City, Nigeria. \\ ${ }^{3}$ Department of Microbiology, Faculty of Life Sciences, University of Benin, Benin City, Nigeria. \\ E-mail: richyomos@yahoo.com
}

\begin{abstract}
Forty two consecutive clinical isolates of Pseudomonas aeruginosa were screened for the presence of reserpine inhibited multidrug (MDR) efflux pump, utilizing ciprofloxacin ofloxacin and/or pefloxacin as substrates, by determining the minimum inhibitory concentration in the presence and absence of $100 \mathrm{mg} / \mathrm{L}$ reserpine. The result showed that $50 \%$ of the Pseudomonas aeruginosa isolates possessed reserpine inhibited MDR efflux pump. MDR efflux pump requiring ofloxacin $(40.48 \%)$ were significantly $(p<0.01)$ more among the isolates when compared with those requiring ciprofloxacin $(16.67 \%)$ or pefloxacin $(11.90 \%)$. Only one isolate possessed reserpine inhibited MDR efflux pumps that utilize all three fluiroquinolones. Research into suitable combination of antibacterials and appropriate pump mactivators or antibacterials that are less likely to be substrate for MDR pumps is advocated.
\end{abstract}

Keywords: Efflux pump, Reserpine, Fluoroquinolones, Pseudomones aeruginosa

\section{INTRODUCTION}

Efflux pumps are transport proteins involved in the extrusion of toxic substrates (including virtually all classes of clinically relevant antibiotics) from within cells into the external environment (Webber and Piddock, 2003). They are found in both procaryotes and eucaryotes (Bambeke et al., 2003). Gram-negative bacteria are generally much more resistant than Grampositive bacteria to a variety of antimicrobial agents (Li et al., 2000). In the case of Pseudomonas aeruginosa, this has traditionally been attributed to the presence of a highly impermeable outer membrane (OM) (Nikaido, 1989). However, recent reports attribute synergy between low OM permeability and broadly specific drug efflux pumps to be responsible for Pseudomonsa aeruginosa intrinsic resistance ( $\mathrm{Li}$ et al., 2000; Zhang et al., 2001; Chuanchuen et al., 2002). Efflux pump can result in both intrinsic and acquired multidrug (MDR) resistance (Nikaido, 1998; Poole, 2000). Several MDR efflux systems have been described in Pseudomonas aeruginosa, including the MexAB-OprM, MexCD-OprJ, MexEF-OprN, MexXY-OprM and MexJK-OprM (Li et al., 2000; Chuanchuen et al., 2002).

The susceptibility of Pseudomonas aeruginosa strains to antimicrobial agents can be significantly enhanced by pump inactivation (Li et al., 1995) and reserpine - a plant alkaloid, has been used to inactivate MDR efflux pump (Neyfakh et al., 1993; Schmctz et al., 1998; Ribera et al., 2002). The importance of detecting MDR efflux pump is to provide information for research into new drugs, coupling existing drugs to pump inactivators or modify existing drugs to make them less likely to be substrate for MDR efflux pump. A lot of report on MDR efflux pumps form various parts of the world exist, but there are none (to our knowledge) from Nigeria. Hence this study focuses on the prevalence reserpine inhibited MDR efflux pump of Pseudomonas aeruginosa to three most commonly prescribed fluoroquinolone in Nigeria.

\section{MATERIALS AND METHODS}

\section{Bacterial Isolates}

Forty two consecutive clinical isolates of Pseudomonsa aeruginosa obtained from the Medical Microbiology Department, University of Benin Teaching Hospital, Benin City, Nigeria, were used for this study. An isolate was idenfied as Pseudomonsa aeruginosa if it was a Gram-negative bacillus, oxidase positive, non-lactose fermenting motile, grows on $0.03 \%$ centrimide agar, production of pyocyarin on nutrient agar after $24 \mathrm{~h}$ incubation at $37^{\circ} \mathrm{C}$ and growth at $42{ }^{\circ} \mathrm{C}$.

\section{Determination of Minimum Inhibitory Concentration (MIC)}

Minimum Inhibitory Concentration (MIC) of ciprofloxacin (V.S. International PVT Ltd, India) ofloxacin (Nigeria German Chemicals, Nigeria) and pefloxacin (Rhone Poulene, France) against the clinical isolates of Pseudomonas aeruginosa were determined using the two - fold serial broth (Muelier -Hinton Broth - Control S, Spain) dilution method with an inculum of $1 \times 10^{6}$ cells $/ \mathrm{mL}$. All experiments were repeated three times with 
Mal. J. Microbiol. Vol 3(2) 2007, pp.37-40

and without $100 \mathrm{mg} / \mathrm{L}$ reserpine $(\mathrm{BDH}$ Chemicals Ltd, England). The MIC was taken as the lowest concentration inhibiting visible growth after $18 \mathrm{~h}$ incubations at $37^{\circ} \mathrm{C}$.
Reserpine inhibited MDR effelux pump was inferred if the MIC with reserpine was four-fold or lower than the MIC without reserpine.

Chi $\left(X^{2}\right)$ square test was used, manually, to determine significant difference.

Table 1: The effect of reserpine on the MIC some fluorquinolones

\begin{tabular}{|c|c|c|c|c|c|c|}
\hline \multirow[t]{2}{*}{ Strain number } & \multicolumn{6}{|c|}{ Pseudomonas aeruginosa MIC ( $\mathrm{mg} / \mathrm{L})$} \\
\hline & CIP & $\mathrm{CIP}^{\mathrm{a}}$ & OFX & OFX $^{a}$ & PER & PEF $^{\text {a }}$ \\
\hline 1 & 320 & 80 & 640 & 160 & 640 & 320 \\
\hline 2 & 0.0391 & 0.0391 & 20 & 0.0195 & 0.0781 & 0.0781 \\
\hline 3 & 40 & 20 & 160 & 80 & 640 & 640 \\
\hline 4 & 1.25 & 1.25 & 2.5 & 2.5 & 0.625 & 0.3125 \\
\hline 5 & 40 & 40 & 160 & 40 & 80 & 40 \\
\hline 6 & 40 & 40 & 320 & 80 & 640 & 320 \\
\hline 7 & 640 & 320 & 640 & 640 & 640 & 320 \\
\hline 8 & 40 & 20 & 320 & 80 & 320 & 320 \\
\hline 9 & 20 & 10 & 160 & 80 & 320 & 160 \\
\hline 10 & 10 & 0.0781 & 10 & 10 & 40 & 40 \\
\hline 11 & 640 & 320 & 80 & 10 & 640 & 20 \\
\hline 12 & 5 & 2.5 & 640 & 10 & 20 & 20 \\
\hline 13 & 320 & 320 & 640 & 640 & 640 & 640 \\
\hline 14 & 0.1563 & 0.1563 & 0.625 & 0.625 & 0.625 & 0.625 \\
\hline 15 & 320 & 160 & 640 & 640 & 640 & 640 \\
\hline 16 & 10 & 0.0391 & 0.625 & 0.625 & 0.625 & 0.625 \\
\hline 17 & 10 & 0.0195 & 10 & 10 & 40 & 0.0195 \\
\hline 18 & 5 & 5 & 80 & 10 & 640 & 10 \\
\hline 19 & 0.0195 & 0.0195 & 320 & 10 & 2.5 & 2.5 \\
\hline 20 & 0.0391 & 0.0391 & 1.25 & 0.625 & 2.5 & 2.5 \\
\hline 21 & 40 & 40 & 320 & 0.0391 & 640 & 640 \\
\hline 22 & 40 & 40 & 320 & 320 & 640 & 640 \\
\hline 23 & 320 & 160 & 640 & 320 & 640 & 640 \\
\hline 24 & 40 & 40 & 640 & 320 & 320 & 320 \\
\hline 25 & 80 & 40 & 80 & 80 & 320 & 320 \\
\hline 26 & 640 & 640 & 640 & 640 & 640 & 640 \\
\hline 27 & 80 & 80 & 160 & 80 & 320 & 320 \\
\hline 28 & 80 & 40 & 320 & 160 & 320 & 320 \\
\hline 29 & 80 & 20 & 320 & 80 & 320 & 320 \\
\hline 30 & 640 & 320 & 320 & 80 & 640 & 320 \\
\hline 31 & 320 & 320 & 640 & 80 & 640 & 640 \\
\hline 32 & 320 & 10 & 320 & 160 & 160 & 80 \\
\hline 33 & 40 & 0.3125 & 80 & 20 & 320 & 40 \\
\hline 34 & 640 & 640 & 320 & 320 & 320 & 320 \\
\hline 35 & 2.5 & 2.5 & 1.25 & 1.25 & 0.3125 & 0.3125 \\
\hline 36 & 20 & 10 & 80 & 80 & 80 & 40 \\
\hline 37 & 0.1563 & 0.1563 & 10 & 0.1563 & 0.1563 & 0.1563 \\
\hline 38 & 0.1563 & 0.1563 & 0.1563 & 0.1563 & 0.1563 & 0.1563 \\
\hline 39 & 0.1563 & 0.1563 & 0.1563 & 0.1563 & 0.1563 & 0.1563 \\
\hline 40 & 0.625 & 0.3125 & 0.625 & 0.625 & 0.625 & 0.625 \\
\hline 41 & 20 & 10 & 20 & 0.0391 & 160 & 40 \\
\hline 42 & 320 & 160 & 80 & 20 & 320 & 320 \\
\hline
\end{tabular}

Numbers in bold are MICs affected by reserpine by four - fold or more. CIP, Ciprofloxacin, OFX, Ofloxacin; PEF, Pefloxacin

${ }^{\mathrm{a}} \mathrm{MIC}$ of Fluoroquinolone in the presence of reserpine. 


\section{RESULTS AND DISCUSSION}

The presence of reserpine inhibited MDR efflux pump was detected in $21(50 \%)$ of the 42 clinical isolates of Pseudomonas aeruginosa (Table 1). Reserpine has been shown not to inhibit all types of efflux pumps (Ribera et al., 2002). Also, it has been reported that strains of Staphylococcus aureus very in the extent to which reserpine is able to block Nor A or other pumps (Schmitz et al., 1998) and this is as a result of reserpine resistance, as has been observed in Bacillus subtilis (Ahmed et al., 1993). These may partly explain the absence of reserpine MDR inhibited efflux pumps in some strains of Pseudomonsa aeruginosa. The other possible reasons include absence of reserpine inhibited pumps, and for the resistant strains, resistance may be due to other mechanisms such as decrease outer membrane (OM) permeability, mutation resulting in altered target site with less affinity for the drugs.

Only one isolate (2.38\%) utilize all three fluorquinolones as substrate for efflux pump while $6(14.29 \%)$ utilize any two of the three fluoroquinolones (Table 1). Of the 6 isolates, 2 utilize ciprofloxacin and ofloxacin; 1 utilizes ciprofloxacin and pefloxacin while 3 utilize ofloxacin and pefloxacin as substrates. This further confirms multiple efflux pumps in Pseudomonas aeruginosa and indeed this organism possess several ( $\mathrm{Li}$ et al., 2000).

The prevalence of reserpine inhibited MDR efflux pump requiring ofloxacin as substrate $(40.48 \%)$ was significantly $(P<0.01)$ higher than that requiring either ciprofloxacin $(16.67 \%)$ or pefloxacin $(11.90 \%)$ as substrate (Table 2). This indicates that resistance to ofloxacin, in comparison with the other two fluoroquinolones used, is most likely due to reserpine inhibited MDR efflux pump.

The MIC of ciprofloxacin in reserpine inhibited MDR efflux pump Pseudomonsa aeruginosa strains decrease by a range of $4-512$ fold with $4(57.14 \%)$ of the 7 strains exhibiting efflux pump becoming susceptible [using MIC breakpoint of $\leq 1 \mathrm{mg} / \mathrm{L}$ according to Andrews (2004)]; ofloxacin MIC decreased by a range of $4-8192$ fold with $4(23.53 \%)$ of the 17 strains exhibiting efflux pump becoming susceptible [using MIC breakpoint of $\leq 2 \mathrm{mg} / \mathrm{L}$ according to Andrews (2004)] and for pefloxacin it decrease by a range of $4-2048$ fold with $1(20 \%)$ of the 5 strains becoming susceptible [no available breakpoint was found, but that for ofloxacin was used]. The result suggests that efflux pump activity in Pseudomonsa aeruginosa strains, functions at various levels. This has also been suggested in Staphylococcus aureus (Kaatz and Seo, 1995; Schmitz et al., 1998).

Combination of any of the studied fluoroquinolomes with an MDR efflux pump inhibitor, such as reserpine, may be effective in the treatment of infections caused by resistant Pseudomonosa aeruginosa stains exhibiting MDR efflux pump. However, reserpine also affects eucaryotes and this makes it unsuitable (Bambeke, et al., 2003). Thus, research should focus on new and safer inhibitors. Also, drugs that are less likely to be substrates for MDR efflux pumps should be developed.

Conclusively, $50 \%$ of the clinical isolates of Pseudomonsa aeruginosa used in this study, possess reserpine inhibited MDR efflux pump and ofloxacin was the drug most likely to be utilized as substrates.

Table 2: Prevalence of reserpine inhibited efflux pump

\begin{tabular}{lll}
\hline Fluroquinolones & $\begin{array}{l}\text { Number } \\
\text { tested }\end{array}$ & $\begin{array}{l}\text { Number with } \\
\text { inhibited efflux } \\
\text { pump (\%) }\end{array}$ \\
\hline Ciprofloxacin & 42 & $7(16.67)$ \\
Ofloxacin & 42 & $17(40.48)$ \\
Pefloxacin & 42 & $5(11.90)$ \\
\hline
\end{tabular}

$X^{2}=9.4267, p<0.01$

\section{REFERENCES}

Ahmed, M., Borsch, C.M., Neyfakh, A.A. and Schuldiner, S. (1993). Mutants of the Bacillus subtilis multidrug transporter Bmr with altered sensitively to the antihypertensive alkaloid reserpine. Journal of Biological Chemistry 268: 11086 - 11089.

Andrews, J.M. (2004). BSAC standardized disc susceptibility testing method (version 3). Journal of Antimicrobial Chemotherapy 53: 713 - 728.

Bambeke, V.F., Glupczynski, Y., Plesiat, Pechere, J.C. and Tulkens, P.M. (2003). Antibiotic efflux pumps in prokaryotic cells: occurrence, impact on resistance and strategies for the future of antimicrobial therapy. Journal of Antimicrobial Chemotherapy 51: 1055 1065.

Chuanchuen, R., Narasaki, C.T. and Schweizer, H.P. (2002). The MexJk efflux pump of Pseudomonas aeruginosa requires OprM for antibiotic efflux but not for triclosan. Journal of Bacteriology 184 (18): 5036 $-5044$.

Kaatz, G.W. and Seo, S.M. (1995). Inducible nor Amediated multidrug resistance in staphylococcus aureus. Antimicrobial agents and Chemotherapy 39: 2650 - 2655.

Li X. - Z., Nikaido, H. and Poole, K. (1995). Role of mexA-MexB-pprM in antibiotic efflux in Pseudomonas aeruginosa. Antimicrobial Agents and Chemotherapy 39: 1984 - 1953.

Li, X - Z., Zhang, L. and Poole, K (2000). Interplay between the mexA-mexB-OprM multidrug efflux antibiotic resitance of Pseudomonas aeruginosa. Journal of Antimicrobial Chemotheraphy 45: 433 436.

Neyfakh, A.A., Borsch, C.M. and Kaatz, G.W (1993). Fluoroquinolone resistance protein Nor $A$ of Staphylococcus aureus is a multidrug efflux transporter. Antimicrobial Agents and Chemotherapy 37: 128 - 129. 
Nikaido, H. (1989). Outer membrane barrier as a mechanism of antimicrobial resistance. Antimicrobial Agents and Chemotherapy 33: 1831 - 1836.

Nikaido, H. (1998). The role of outer membrane and efflux pumps in the resistance of Gram-negative bacteria. Can we improve drug access? Drug Resistance update 1: 93 - 98.

Poole, K. (2002). Efflux-mediated resistance to fluoroquinolones in Gram-negative bacteria. Antimicrobial Agents and Chemotheraphy 44: 2233 $-2241$.

Ribera, A., Ruiz, J., Jimenez de Anta, M. T. and Vila, J. (2002). Effect of efflux pump inhibitor on the MIC of nalidixic acid for Acinetobacter baumanii and Stenotrophomonas maltophilia clinical isolates. Journal of Antimicrobial Chemotherapy 49: 697 698.

Schmitz, F.-J., Fluit, A.C., Luckefahr, M., Engler, B., Hofmann, B., Verhoef, J., Heinz, H.-P., Hadding, $U$. and Jones, M.E. (1998). The effect of reserpine, an inhibitor of multidrug efflux pumps, on the in-vitro activities of ciprofloxacin, sparfloxacin, and moxifloxacin against clinical isolates of Staphlococcus aureus. Journal of Antimicrobial Chemotheraphy 42: 807 - 810.

Webber, M.A. and Piddock, L.J.V. (2003). The importance of efflux pumps in bacterial antibiotic resistance. Journal of Antimicrobial Chemotherapy 51: 9 - 11.

Zhang, L., Li, X.-Z. and Poole, K (2001). Fluoroquinolone susceptibilities of efflux - mediated multidrug resistant Pseudononas aeruginosa, Stenotrophmonas maltophila and Burkholderia cepacia. Journal of Antimicrobial Chemotherapy 48: 549 - 552. 